\title{
Identifying Return Distribution of Sri Lankan Stock Market Index
}

\author{
N. V. Chandrasekara ${ }^{1, *}$, C. D. Tilakaratne ${ }^{2}$ \\ ${ }^{1}$ University of Kelaniya, Sri Lanka \\ ${ }^{2}$ University of Colombo, Sri Lanka \\ *Corresponding Author: nvchandrasekara@kln.ac.lk
}

Copyright (C 2014 Horizon Research Publishing All rights reserved.

\begin{abstract}
In the current financial world, prediction of stock returns has become a vital task. Many prediction techniques available recently depend on the return distribution of stock index. Identifying return distribution of stock return has an immense interest among researchers nowadays. Many researchers have proposed different distributions to model the return distribution of stock market indices. However a study aimed at finding the distribution of return series of local stock indices was not found. In this study return distributions of All Share Price Index (ASPI) of the Colombo stock exchange was examined. The study period consists of 5 years daily data from 1st August 2007 to 31 st July 2012 of the ASPI. Results display that the return distribution of ASPI cannot be modeled using Normal distribution and Student's $t$ distribution. The Scaled $t$ distribution with parameters $\mathrm{mu}=0.0000613719$, sigma $=$ 0.00619983 and $\mathrm{nu}=2.54137$ can be introduced as the best distribution to model the return distributions of All share price index. Kolmogorov-Smirnov (K-S) Test has been used to access the suitability of fitted distribution. Random numbers were generated using Scaled $t$ distribution with above mentioned parameters and the K-S test was carried out using the generated series and the return series of ASPI. The same procedure was repeated 100 times in order to improve the accuracy of results. Minimum p-value of 0.0534 was obtained in the simulation study and exhibit that the test is not significant under 5\% level of significance above $95 \%$ times. Finding of this research will help many researches in the financial sector of Sri Lanka to use an appropriate distribution for modeling the ASPI returns and hence to enhance the forecasting accuracy.
\end{abstract}

Keywords Return Distribution, All Share Price Index, Colombo Stock Exchange, Scaled $\mathrm{T}$ Distribution, Kolmogorov-Smirnov Test

\section{Introduction}

Predictability of financial markets depicts crucial importance in recent world. Stock market exhibits great interest due to profitability and development of many techniques among all financial markets. Many researchers interested in predicting stock market index recently. Finding distribution of stock returns will be useful in building prediction models for stock indices. Therefore, identifying an appropriate distribution to model the return distribution of stock index becomes a vital important factor nowadays.

Over the last few decades many researchers attempt to find a suitable distribution to model stock return distribution. Even though the traditional belief is that the return distribution follows the Normal /Gaussian distribution, many researches provide evidence to say that the return distribution is deviate from the Normal distribution. In 1991 Badrinath and Chatterjee found that the return distributions are elongate from the Gaussian distribution by their research done for the New York Stock Exchange. [10] showed that the daily stock returns display significant departures from normality by examining six stock markets namely New York, Tokyo, London, Paris, Frankfurt, Paris and Madrid. The research done for thirteen European markets and four Scandinavian markets by [1] clearly rejected the normality of stock return distribution. Many other researches provided substantiation to reject the normal distribution to model return distribution of stock market indices such as $[6,7,8,11$, 12].

Since the stock return distribution deviate from the Normal distribution, next approach of the researchers was to find a suitable distribution to model the return distribution of stock market indices. By considering different techniques and different stock markets many researchers have tried to find the stock return distribution.

The research carried out by Doric and Doric in the year 2011 to find the adequate return distribution of Belgrade Stock Exchange suggested that the Student's t distribution and Normal Inverse Gaussian distribution are acceptable to model the return distribution of stock indices.

A study done by considering six stock markets by [10], recommended Scaled t distribution as the best distribution to model return distribution of all considered markets by rejecting several other distributions such as Normal, Paretian, 
Logistic, Student's t, exponential power and discrete mixture of two normal. Scaled t distribution was introduced as the best distribution to model return distribution by [1] in their study which considered thirteen European markets. Same researchers have done a similar study for four Scandinavian markets and proved the same result. i.e. the Scaled $t$ distribution is well fitted to return distribution, by rejecting Logistic distribution and exponential power distribution. By considering S\&P 500 index [7] provided the fact that the Scaled t distribution can be used to model return distribution accurately.

Although literature provides evidence regarding different distribution to model stock return distribution of many stock indices, no evidence was found about a study which aimed at finding the distribution of returns of the local stock indices. Therefore, finding the suitable distribution to model All Share Price Index (ASPI) of Colombo Stock Exchange which is the objective of this research is a timely need. This task can be commenced by considering the distributions recommended by other researchers to model return distribution of different stock indices. Finding of the most suitable distribution to model ASPI index will help many researches in the financial sector of Sri Lanka to use an appropriate distribution instead of Normal distribution for modeling the ASPI returns and hence to enhance the forecasting accuracy.

The rest of the article is organized as follows: In the next section, the data and techniques useful for the study are described. Results of the study and discussion are demonstrated subsequently. Then the conclusion is provided and references conclude the article.

\section{Methodology}

\subsection{Data Collection and Data Pre-Processing}

Daily Closed Price of All Share Price Index for five years period from $1^{\text {st }}$ August 2007 to $31^{\text {st }}$ July 2012 was considered in this study. As a proved mechanism, holidays were filled with previous day's value in the series and the data series covers 1290 observations.

The Following formula was used to calculate the daily returns of the market index:

$$
\mathrm{R}_{\mathrm{t}}=\left(\mathrm{P}_{\mathrm{t}}-\mathrm{P}_{\mathrm{t}-1}\right) / \mathrm{P}_{\mathrm{t}-1}
$$

where $R_{t}$ - return of the day $t, P_{t}$ closed stock price of the day $t$ and $P_{t-1}$ - closed stock price of the day $t-1$.

\subsection{Normal Distribution}

The normal distribution is symmetric bell shaped curve which is widely used and important statistical distribution. Many natural phenomena can be modeled using the normal distribution. Normal distribution or the Gaussian distribution is a continuous distribution with following probability density function (p.d.f.):

$$
f(x)=\frac{1}{\sigma \sqrt{2 \pi}} e^{-\frac{(x-\mu)^{2}}{2 \sigma^{2}}}
$$

where $\mu$ is the mean or expectation of the distribution and $\sigma$ is the standard deviation (Mood, Graybill and Boes (1974)).

\subsection{Student's t Distribution}

The $t$ distribution is a continuous probability distribution with one parameter called degrees of freedom. The probability density function of Student's $t$ distribution is displayed in Equation 3.

$$
\frac{\Gamma\left(\frac{\nu+1}{2}\right)}{\sqrt{\nu \pi} \Gamma\left(\frac{\nu}{2}\right)}\left(1+\frac{x^{2}}{\nu}\right)^{-\frac{\nu+1}{2}}
$$

where $v$ is the degrees of freedom and $\Gamma(\bullet)$ represent the gamma function (Mood, Graybill and Boes (1974)).

\subsection{Scaled t Distribution}

The Scaled $t$ distribution is useful for modeling data distributions with heavier tails which has three parameters namely location, scale and shape. Smaller values of the shape parameter yield heavier tails. The probability density function of the Scaled t distribution is as follows:

$$
f(x)=\frac{\Gamma\left(\frac{v+1}{2}\right)}{\sqrt{\pi(v-2) \sigma^{2}} \Gamma\left(\frac{v}{2}\right)}\left[1+\frac{(x-\mu)^{2}}{(v-2) \sigma^{2}}\right]^{-\left(\frac{v+1}{2}\right)}
$$

where $\Gamma(\bullet)$ represent the gamma function, $\mu$ is the location parameter, $\sigma$ is the scale parameter and $v$ is the shape parameter [1].

\subsection{Quantile-Quantile Plot (Q-Q Plot)}

The Quantile-Quantile plot can be used to determine whether two data sets come from populations with a common distribution. In this graphical technique, quantiles of the first data set against the quantiles of the second data set is plotted and a 45-degree reference line is used to interpret. If the two data sets come from a population with the same distribution the points should fall approximately along this reference line. The greater the departure from this reference line, the greater the evidence for the conclusion that the two data sets have come from populations with different distributions. This technique can provide an assessment of "goodness of fit" that is graphical and more powerful approach than the common technique of comparing histograms of the two samples [4].

\subsection{Kolmogorov-Smirnov Test (K-S test)}

The Kolmogorov-Smirnov test is a nonparametric test which is used to assess the equality of continuous, one-dimensional probability distributions. In one-sample $\mathrm{K}-\mathrm{S}$ test, a comparison of a sample with a reference probability distribution is preformed and the null hypothesis is that the sample is drawn from the reference distribution. 
The Kolmogorov-Smirnov statistic quantifies a distance between the empirical distribution function of the sample and the cumulative distribution function of the reference distribution in one sample test. Comparison of the two samples is performed in two-sample K-S test under the null hypothesis that the two samples are drawn from the same distribution. In two sample test K-S statistic quantifies a distance between the empirical distribution functions of two samples. In each case, the distributions considered under the null hypothesis are continuous distributions.

The two-sample Kolmogorov-Smirnov test is one of the most useful and general nonparametric methods for comparing two samples. The following null and the alternative hypothesis are used in the two sample K-S test.

$\mathrm{H}_{0}$ : Two samples are drawn from the same distribution

$\mathrm{H}_{1}$ : Two samples are not drawn from the same distribution

The Kolmogorov-Smirnov statistic for two-sample test is:

$$
D_{n . n^{\prime}}=\sup _{x}\left|F_{1, n}(x)-F_{2, n^{\prime}}(x)\right|
$$

where $\sup _{x}$ is the supreme of the set of distances and $F_{1, n}$ and $F_{2, n^{\prime}}$ are the empirical distribution functions of the first and the second sample respectively.

The null hypothesis is rejected at level $\alpha$ (which is the significance level) if, $D_{n, n^{\prime}}>c(\alpha) \sqrt{\frac{n+n^{\prime}}{n n^{\prime}}}$ where the value of $c(\alpha)$ is given in standard statistical tables [5].

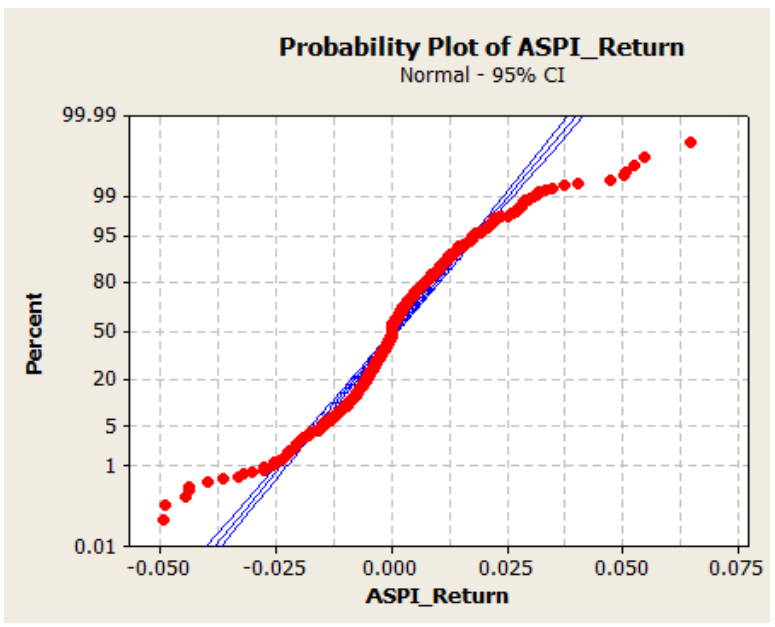

Figure 1. Normal probability plot of ASPI

\section{Results and Discussions}

Even though evidence from literature suggests that the stock return distribution cannot be modeled using Normal distribution, Normal probability plot was drawn to the ASPI return series to check the normality and displayed below.

Figure 1 exhibits that the normal probability plot of ASPI deviates from the straight line. Therefore it is evident that the normal distribution cannot be used to model the return distribution of All Share Price Index.

Student's t distribution was fitted to the return series of the
ASPI and check the adequacy using Kolmogorov-Smirnov test. Numerous tests were carried out by changing parameters of the distribution. Results indicate that the p-values obtained in all the cases are less than 0.05 and the test is significant under $5 \%$ level of significance in all the cases. Therefore it can be concluded that Student's $t$ distribution is also not suitable to model the return distribution of all share price index of Colombo stock exchange.

With the evidence from literature, Scaled $t$ distribution was fitted to the return series of ASPI and calculated the parameters of the distribution. Suitability of the fitted distribution was assessed using Q-Q plot and Kolmogorov-Smirnov test. Figure 2 illustrates the fitted Scaled $t$ distribution for the return series of the ASPI.

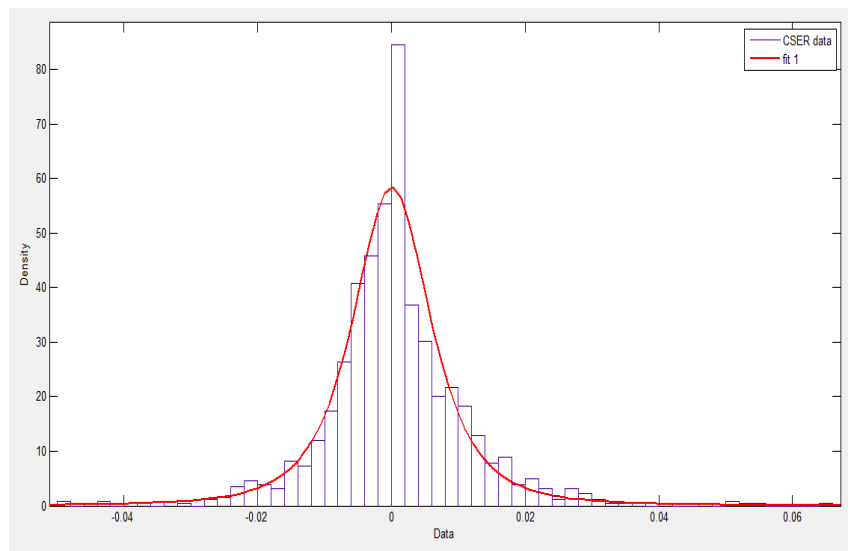

Figure 2. Fitted Scaled t distribution for ASPI return series

Figure 2 shows that scaled $t$ distribution exhibits a good fit to the return distribution of the ASPI. Three parameters are associated with the Scaled $t$ distribution namely location parameter $(\mathrm{mu})$, scale parameter (sigma) and shape parameter $(n u)$. Estimated parameters for fitted Scaled $\mathrm{t}$ distributions for the ASPI series are represented in Table1.

Table 1. Estimated parameters of the fitted Scaled $t$ distributions for ASPI return series

\begin{tabular}{|c|c|c|c|}
\cline { 2 - 4 } \multicolumn{1}{c|}{} & \multicolumn{3}{c|}{ Parameter Estimates } \\
\hline $\begin{array}{c}\text { Return } \\
\text { Series }\end{array}$ & $m u$ & sigma & $n u$ \\
\hline ASPI & 0.0000613719 & 0.00619983 & 2.54137 \\
\hline
\end{tabular}

The shape parameter $n u$ of the Scaled t distribution can capture the heaviness of tails in the fitted distribution. A smaller $n u$ indicates heavy tails. Since it is a known fact that the return distribution of stock market indices exhibit heavy tails, estimated values for $n u$ which are small lead to consider that the heavy tails are well captured by the Scaled $t$ distribution in the return series.

The following figure illustrates the Q-Q plot drawn to evaluate the fitted Scaled $t$ distribution graphically.

In Figure 3, Q-Q plot demonstrate linear patterns which confirms that the two distributions considered in graph are similar. Therefore, it can be said that the fitted Scaled $\mathrm{t}$ 
distributions with respective parameters exhibit a good fit for the return distribution ASPI index.

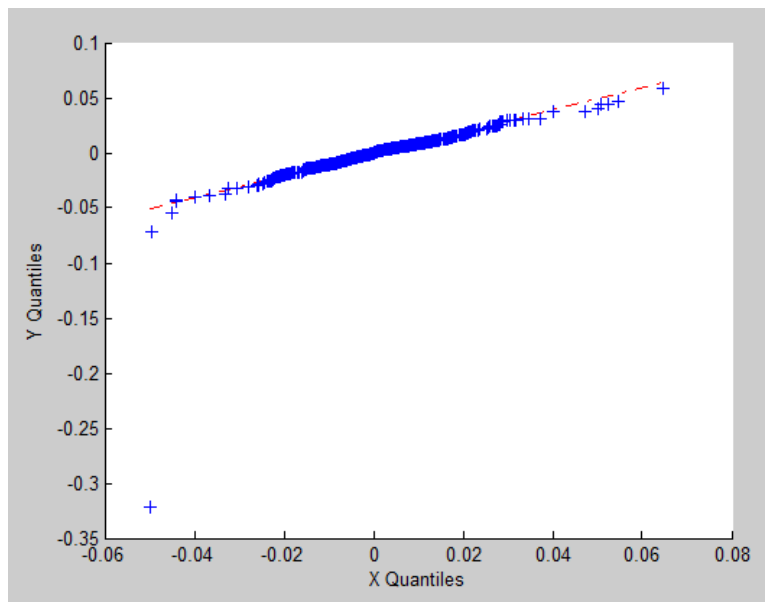

Figure 3. Q-Q plot for ASPI return series

Kolmogorov-Smirnov Goodness-of-Fit test was used to assess the adequacy of fitted Scaled $t$ distributions for return series and p-values were calculated. Random numbers were generated using Scaled $t$ distribution with above mentioned parameters and the K-S test was carried out using the generated series and the return series of ASPI. The same procedure was repeated 100 times in order to improve the accuracy of results. Minimum p-value of 0.0534 was obtained in the simulation study. Results indicated that the test is not significant under 5\% level of significance above $95 \%$ times. Therefore it can be said that the Scaled $t$ distribution with parameters $m u=0.0000613719$, sigma $=$ 0.00619983 and $n u=2.54137$ can model the return series of ASPI index very well. This result will be useful for further research in constructing models to predict the ASPI of Colombo Stock Exchange.

The main drawback of this research is that the study period considered is only for five years which is a short period and consequently with a longer study period the findings may be different.

\section{Conclusions}

Return distributions of the ASPI is deviate from the Normal distribution and Student's $\mathrm{t}$ distribution. Scaled $\mathrm{t}$ distribution can be recommended as the best distribution to model the return distribution of the All Share Price Index of
Colombo Stock Exchange.

\section{REFERENCES}

[1] Aparicio, F. \& Estrada, J. (1997). Empirical Distributions of Stock Returns: European Securities Markets, 1990-95. Business Economics Series 02, Universidad Carlos de la Empresa, Spain.

[2] Aparico, F. \& Estrada, J. (1997). Empirical Distributions of Stock Returns: Scandinavian Securities Markets, 1990-95. Statistics and Economics Series of Universidad Carlos III de Madrid, 96, 58.

[3] Badrinath, S.G. \& Chatterjee, S. (1991). A Data Analytic look at skewness and elongation in common -stock-return distributions. Amarican Statistical Association Journal of Economic Statistics, 9. 2, 223-233.

[4] Beirlant, J., Geogebeur, Y., Segers, J. \& Teugels, J. (2004). Statistics of Extremes: Theory and Applications. England. . John Wilay \& Sons Ltd.

[5] Conover, W. J. (1999). Practical Non parametric Statistics. United States of America. John Wilay \& Sons, Inc.

[6] Doric, D. \& Doric, E.N. (2011). Return Distribution and Value at Risk Estimation for BELEX15. Journal of Operations Research, 21, 103-118.

[7] Egan, W.J. (2007). The Distribution of S\&P Index Returns. Unpublished research report. papers.ssrn.com.

[8] Fortin, I. \& Kuzmics, C. (2002). Tail-Dependence in Stock-Return Pairs. Economics Series of Institute for Advanced Studies, Vienna. 126.

[9] Mood, A. M., Graybill, F. \& Boes, D. C. (1974). Introduction to the theory of statistics. Singapore : Mc-Graw-Hill.

[10] Peiro, A. (1994). The Distribution of Stock Returns: international evidence. Applied Financial Economics, 4, 431-439.

[11] Rachev, S.T., Emeritus, Stoyanov, S.V., Wu, C. \& Fabozzi, F.J. (2007). Empirical Analyses of Industry Stock Index Return Distributions for Taiwan Stock Exchange. Analysis of Economics and Finance, 8, 1, 21-31.

[12] Wang, B.H. \& Hui, P.M. (2001). The Distribution and Scaling of fluctuations for Hang Seng index in Hong Kong stock market. The European Physical Journal B, 20, 573-579. 